\title{
IX. Magnetic rotation in iron cathode films
}

\section{L.R. Ingersoll Ph.D.}

To cite this article: L.R. Ingersoll Ph.D. (1909) IX. Magnetic rotation in iron cathode films , Philosophical Magazine Series 6, 18:103, 74-102, DOI: 10.1080/14786440708636674

To link to this article: http://dx.doi.org/10.1080/14786440708636674

册 Published online: 21 Apr 2009.

Submit your article to this journal 정

Џll Article views: 2

Q View related articles ¿

Citing articles: 13 View citing articles 
The power supplied by the source is

$$
\begin{aligned}
& =\frac{1}{2} \cdot \frac{\omega m}{2} \overline{\mathrm{OE}^{\prime}} \cdot \overline{\mathrm{a}}_{1} \cos \mathrm{a}_{1} \mathrm{O \textrm {w } ^ { \prime }}, \\
& =\frac{\omega m}{4} \overline{\mathrm{a}}_{1} \bar{\kappa} \sin \mathrm{a}_{1} \mathrm{O} \kappa, \\
& =\frac{\omega m}{2} \times \text { area of triangle } \mathrm{a}_{1} \mathrm{O} \kappa,
\end{aligned}
$$

and the power developed by the motor $=\omega \mathrm{T}=\frac{\omega m}{2} \times$ sum of areas of the triangles $\alpha_{0} \mathrm{Oa}_{1}, a_{1} \mathrm{O}_{2}, \alpha_{2} \mathrm{Oa}_{3}, \& \mathrm{c}$, attending to signs.

Hence the efficiency is equal to

$$
\frac{\alpha_{0} O a_{1}+a_{1} \mathrm{O} \alpha_{2}+\alpha_{2} \mathrm{Oa}_{3}+\& \mathrm{c} .}{a_{1} \mathrm{O} \kappa} \text {. }
$$

By rotating the vector from $O$ to the middle point of $\alpha_{0} \alpha_{2}$ backwards through $90^{\circ}$ and doubling we obtain $\mathrm{OE}_{1}$, which represents in amplitude and phase $2 / m \omega$ times the first harmonic of the total E.M.F. of the motor (see $\S 13$ ).

This vector can now be compared with $\mathrm{OE}^{\prime}$ which represents $2 /$ mo times the applied E.M.F.

Fig. 4 easily explains how, by increasing the exciting current of an A.C. motor, the phase of the armature current is advanced relative to that of the applied E.M.F.

IX. Magnetic Rotation in Iron Cathode Films. By L. R. Ingersold, Ph.D., Assistant Professor of Physics, University of Wisconsin*.

[Plate I.]

Part I. The Bolometric Method of Measuring Rotations.

Part II. Rotation dispersion Curves for the Faraday and Kerr Effects in the Infra-red Spectrum.

Part III. Dependence of the Kerr Rotation on the Refractive Index of the Overlying Medium.

\section{INTRODUCTION.}

7 HE purpose of the present work has heen an experimental 1 study of certain problems in the magneto-optics of the magnetic metais, viz. the Faraday and Kerr effects for a long range of spectrum; the thickness of the surface layer which

* Communicated by the Author. This work has been aided by a grant from the Rumford Fund of the American Academy of Arts and Sciences, for which the writer wishes to express his appreciation. 
gives rise to the Kerr rotation; and the relation between the Kerr rotation and the optical density of the surrounding medium. These questions have been studied for the simplest typical case, - that of iron in the form of thin films, the cathode film being chosen for reasons which will appear later.

The rotation of plane polarized light on transmission through thin metallic films in a magnetic field has been studied by Kundt*, Dn Bois $\dagger$, Lobach $\ddagger$, Righi $\$$, Harris $\|$, Skinner and Tool $\Upsilon$, and others. This effect, discovered by $\mathrm{K}$ undt, has since been measured for light of various wavelengths by other investigators, and so-called rotation dispersion curves thus obtained for films of iron, nickel, and cobalt. Recently Harris made some very careful determinations of such curves for iron films deposited cathodically in atmospheres of hydrogen, nitrogen, and oxygen, while Skinner and Tool find that, independent of changes in atmosphere, two different sorts of films with different rotatory powers may be produced for some metals by the cathode discharge, and moreover that the rotation for any wave-length bears more or less relation to the absorption for that wave-length.

An effect quite similar to this of rotation by transmission through a magnetized film, and which, doubtless, owes its existence to quite sinilar if not identical causes, is the socalled Kerr effect, or rotation of the plane of polarization upon normal retlexion from a polished mirror held perpendicular to a strong magnetic field. The mirror may be of iron, cobalt, nickel, or magnetite, and indeed in the earlier experiments was formed by merely polishing one end of a pole-piece of the magnet.

The work of Kerr** was extended by Du Bois ††, who determined the variation of this effect with wave-length for mirrors of the above-mentioned substances. Such rotation dispersion curves resemble those obtained for the case of transmission through thin films, in that they are anomalous, showing in general an increase of rotation with wave-length, as far as the limits of the visible spectrum, instead of a marked decrease, as is the case for practically all transparent

* Wied. Ann. xxiii. p. 228 (1884); xxvii. p. 191 (1886).

$\dagger$ Wied. Ann. xxxi. p. 941 (1887).

I Wied. Ann. xxxix. p. 347 (1890).

§ Mem. R. Accad. Sc. d. Bologna, 1886, p. 443.

il Phys. Rev, xxiv. p. 337 (1907).

If Paper read before the American Physical Society, Chicago, Dec. 1907 .

** Phil. Mag. [5] iii. p. 339 (1877).

t† Wied. Ann. xxxix. p. 25 (1890); Phil. Mag. [5] xxix. p. 253 (1890). 
substances. Moreover, the rotation, while of about the same order of magnitude in the two cases, is opposite in sign, a point not satisfactorily, or at least simply, explained by the theory. Complicated phenomena, which have been studied by many other observers, arise for cases of oblique incidence or of magnetization parallel to the surfice, but in the present work we shall consider only the more common case of nearly normal incidence, and of magnetization perpendicular to the surface.

A few years ago the writer* developed a method of measuring such magnetic rotation for wave-lengths of the infra-red spectrum, and supplemented the work of $\mathrm{Du}$ Bois by extending his curves to about $\lambda=3.5 \mu$. The striking thing shown by these curves was that the rotation in each case reached a maximum for wave-length about $1 \mu$, and decreased rapidly for longer wave-lengths. Moreover, in the case of nickel the rotation changed sign for wave-lengths longer than $1.4 \mu$, while for magnetite the case was still more complicated.

It was desired at the time to proceed at once with measurements on thin films of the magnetic metals, but the determination of such small rotations was too difficult and uncertain a process with the apparatus as it then existed. Accordingly, the method of measurement was considerably modified, and thereby greatly improved, and the apparatus almost entirely rebuilt on a more extensive scale, with a more careful working out of details ; this has made possible the handling of the sort of problems it was desired to take up in this investigation.

Aim of the present work.-This has been : First, to measure the ransmission, or Faraday, rotation for iron films of various thickness for as wide a range of wave-lengths as possible $(\lambda=\cdot 6 \mu$ to $2 \cdot 2 \mu)$, thus supplementing the work of Lobach, Harris, and other's by extending their curves over a spectral region of greater extent. Second, to determine the reflexion rotation, or Kerr effect, for the same films and in the same magnetic field, with the view of correlating as closely as possible these two rotation effects due to transmission and reflexion respectively. Third, to study the dependence of the Kerr rotation on the thickness of the film, a problem suggested by Kundt, and left by him after some preliminary observations with the hope of more careful treatment later. Fourth, to study briefly the effects of oxidation on the forms of the rotation dispersion curves, and the geveral relation between absorbing or reflecting power and rotatory power. Fifth, to * Phil. Mng. [6] lxi. p. 41. (1906). 
investigate the effect on the Kerr rotation of overlaying the surface with liquids of various refractive indices.

These questions are discussed in Parts II. and III. of the present paper. Iron has been chosen as a working substance for these experiments as it gives the largest, and hence most accurately measurable effects, but it is intended in the near future to study part or all of these effects with films of cobalt and nickel as well. The cathode type of film has been used chiefly because it may be readily produced of any desired thickness and without a preliminary deposit of platinum, as would be required for electrolytic films and which would be very objectionable for some of the experiments. It is true that cathode films differ somewhat in optical properties from the massive metal, but the same may be said of the electrolytic film which is well known to be granular in structure. In some cases the cathode film appears to resemble fused metal more nearly than the electrolytic.

\section{Part I.-The Bolometric Method and Apparatus for Measuring Magnetic Rotations.}

This method with the necessary apparatus for measuring rotations for the shorter infra-red wave-lengths has already been described by the writer*, both in its original form and after important modifications had been introduced; but so many changes have been made since then that a brief description of method and apparatus seems desirable here.

The essential difference, in the optical system, between this method and the customary process of measurement applicable to the visible spectrum, arises from the fact that any small rotation of the plane of polarization occurring between crossed nicols, or other polarizing agents, causes the greatest change of intensity when the latter are crossed at $45^{\circ}$, a principle recognized and used over half a century ago by Provostaye and Desainst. 'This, then, is evidently the most effective arrangement when a bolometer or other radiation measuring instrument is to be used instead of the human eye.

In the first arrangement adopted, piles of thin glass plates were used as polarizer and analyser. Light from a Nernst glower, after being polarized by reflexion, passed through the magnet and the rotating substance between its pole-pieces, and after reflexion from the analyser fell on the slit of a

* Phil. Mag. [6] lxi. p. 41 (1906); Phys. Rev. xxiii. p. 489 (1906).

Hereafter referred to as Phil. Mag. loc. cit. and Phys. Rev. loc. cit.

† Ann. Chim. Phys. [3] xxvis. p. 232 (1849). 
spectrometer, to reach finally, after dispersion, the strip of a boloneter. On exciting the magnet the rotation of the polarization plane would cause a change of intensity of the radiation passing the analyser and falling on the bolometer, which change, when divided by the actual intensity of transmitted radiation of any given wave-length, gave a ratio proportional to the rotation for this particular wave-length.

The Compensating Principle.-The foregoing arrangement was capable of yielding very fair results at a great expenditure of labour in observing, but difficulty arose from small and unavoidable changes of brilliancy of the glower which would appear as spurious rotations. After trying many ways of compensating for this, there was finally adopted the scheme of using as analyser a large double-image prism, placed so that each of its principal planes made an angle of $45^{\circ}$ with the original plane of polarization. The two transmitted beams of equal intensity were carried through the spectrometer, and after dispersion were allowed to fall on the two strips of a specially constructed bolometer. Any rotation would thus diminish one beam and increase the other by an equal amount-thus producing a doubled effect-while any small change in brilliancy of the glower would affect each beam equally, and hence give rise to no error. This was immeasurably superior to the former arrangement, and more than made amends for the small loss of range of spectrum its use involved; for the thick calcite prisms (the polarizing plates were also replaced by calcite) would transmit little beyond $\lambda=2 \cdot 5 \mu$.

The very simple mathematics of this method have already been worked out in a former paper ${ }^{*}$, and we need take from this only the formula

$$
\delta=45 / \pi \cdot d \mathrm{I} / \mathrm{I} \text {, }
$$

which gives for any wave-length the rotation $\delta$ in degrees, when $I$ is the intensity of radiation on either bolometer strip for this wave-length, and $d I$ the relative change of intensity of the two beams.

Use of High and Low Dispersion.-One of the problems to be met in this work was that of securing, in spite of the many losses, a sufficiently intense spectrum such that the small changes of intensity amounting to perhaps less than 1/10 per cent. would still be readily measurable. Every effort had been made to secure this in the earlier form of apparatus by the use of large apertures and a brilliant source, hence as a still further increase was desired for the present

$$
\text { * Phys. Rev. loc. cit. }
$$


work, it was necessary to resort to a reduction of dispersion. But this would mean a sacrifice of certainty of wave-length, and would magnify the rather uncertain errors due to the width of the slit image and bolometer strip in the spectrum.

To avoid this two distinct sets of prisms and mirrors were used in connexion with the same spectrometer table, giving in the one case a very intense but correspondingly contracted spectrum, and in the other a spectrum of several fold greater dispersion, but correspondingly weaker in energy.

In practice, then, some film or substance giving a relatively large rotation was tested with each of these combinations, and the two curves compared with a view of determining the effect of the lower dispersion, if any, in distorting the curve. This correction could then be applied with assurance to curves obtained with films which gave such small rotations that only the lower dispersion arrangement could be used.

Tests by Mechanical Rotation.-While in as simple a form as possible, to attain the desired end, the foregoing method is necessarily complicated when compared with the extremely straightforward ways of observing the same phenomena in the visible spectrum. Hence it is desirable to have some way of checking the results, or at least of applying a thorough test. This was done by mounting the polarizer in a divided circle: small and accurately known rotations were then given it, causing of course corresponding rotations of the plane of polarization. These were measured just as if of magnetic origin and checked with the known rotation produced mechanically. The results of this test, as well as of the measurements spoken of in the last paragraph, will be given later.

\section{Apparatus.}

In the matter of general arrangement of apparatus the former order was preserved, as will be seen from fig. 1 . The whole was, however, mounted on massive slate slabs which rested on heavy wooden supports. The component pieces, with the exception of the optical parts, were constructed in the shops of this Department.

Electromagnet.-This had proved so satisfactory since its construction some years ago, that the only change necessary was the addition of cooling coils which carried circulating tap-water, and kept the ends of the pole-pieces at a nearly constant temperature. The method of excitation is worth brief mention. The winding, which consisted of about 6000 feet of No. 10 wire, was grouped in eight coils. The four pole 
coils were most effective, and when excited with 16 amps. at 60 volts, gave a field of about 6000 units, which, while not as high as might be desired - on account of the large air-gap necessary - was very satisfactory for present purposes. Now

Fir. 1.-General arrangement of apparatus.

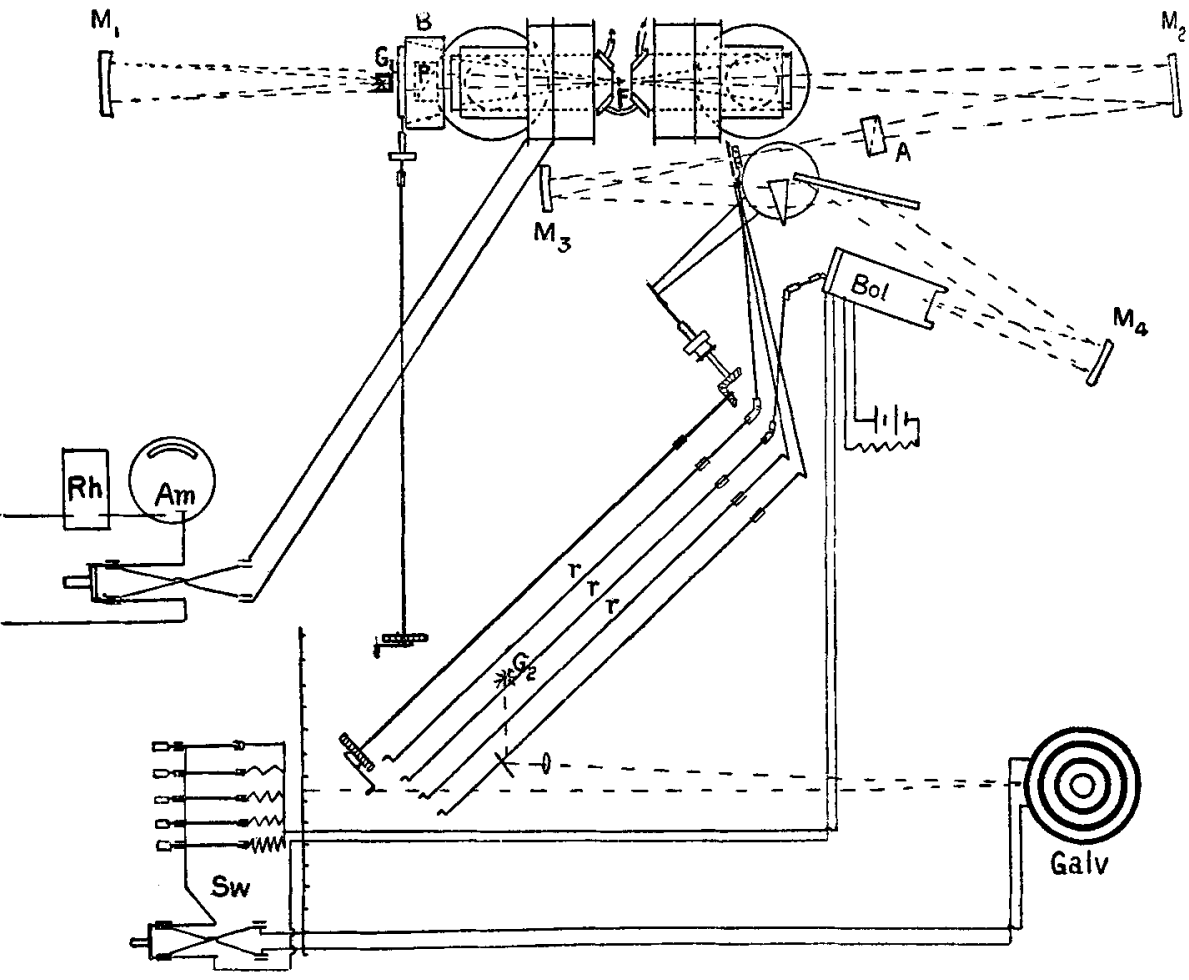

it was very desirable to have as small an external field as possible, particularly at the polarizer which had to be very near the magnet, and this was secured by a combination of series and parallel connexions of the remaining four coils, with suitable resistances, until a compass-needle remained unaffected at the position of the polarizer. This could be done without material sacrifice of the field due to the polecoils.

Optical System.-Light from the D.C. Nernst-glower $\mathrm{G}_{1}$ in its firebrick enclosure was converged by the mirror $\mathbf{M}_{1}$ to an image between the pole-pieces, and to another image on the slit of the spectrometer by the mirror $\mathrm{M}_{2}$. A double image prism $\mathrm{P}$ of $4.5 \mathrm{cms}$. aperture served as polarizer, only 
one of the two beams being used. It was mounted in a massive brass conical bearing $\mathrm{B}$, and could be rotated by a micrometer tangent screw to produce an accurately known mechanical rotation of the plane of polarization, as mentioned above. The analyser, A, was a slightly largeridouble image prism of $5 \mathrm{~cm}$. aperture.

Spectrometer.-The two images (of the glower) from the analyser, each about $1 \mathrm{~cm}$. long, fell vertically over one another on the two parts into which the slit was divided by the diaphragms D, D (fig. 2); and shutters, Sh, allowed

Frg. 2.-The slit and its accessories.

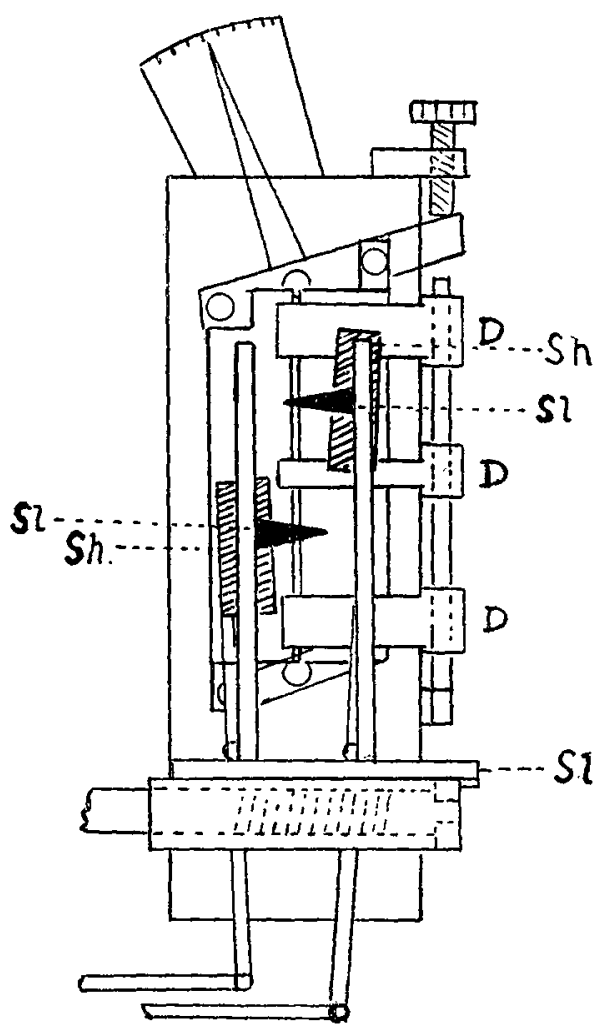

each beam to be cut off separately. The sliding shade, Sl, was necessary for the following reason:-It was required that the initial intensities of the two spectra as they fell on the bolometer strips should be equal. This was accomplished by

Plil. Mag. S. 6. Vol. 18. No. 103. July 1909. 
the two thin wedge-shaped shades, which, when moved across the slits, darkened one image by cutting off a small part of the centre, and at the same time lightened the other until the two beams were of equal intensity. Because of slight inequalities in the bolometer strips this adjustment had to be repeated for each new setting of the spectrometer.

Each of the prisms for the spectrometer was of glass instead of rock-salt as formerly used. There was no need of rocksalt in this case, since there was at best a considerable thickness of glass to be traversed by the light, in connexion with the double image prisms; and, moreover, glass has the alvantage of giving a somewhat more uniform dispersion than rock-salt. The prism of lower dispersive power had a refracting angle of $25^{\circ}$, and was of ordinary flint-glass with faces $9 \times 9 \mathrm{cms}$. It was used with mirrors of $3 \overline{3} \mathrm{cms}$. and $30 \mathrm{cms}$. focns respectively. The otber prism had a $45^{\circ}$ angle and was of extra dense flint with faces $9 \times 7 \cdot 5 \mathrm{cms}$. The corresponding mirrors were of 50 cms. focal length each. This arrangement gave a spectrum from three to four times longer than the other.

Bolometer and Galvanometer.-The former might be said to be of the differential type, as both strips were exposed, being vertically above one another. Each strip was $5 \mathrm{~mm}$. by $8 \mathrm{~mm}$. The balancing coils and slide wire were all selfcontained, and the instrument was remarkably steady and free from drift. The galvanometer, which was of the Thomson astatic type, had a system weighing about $2 \mathrm{mgs}$., and was worked at a sensibility of from 3 to $5 \times 10^{-10} \mathrm{amp}$. per $\mathrm{mm}$. for $6 \mathrm{ohms} \mathrm{resistance.} \mathrm{To} \mathrm{free} \mathrm{it} \mathrm{from} \mathrm{the} \mathrm{influence} \mathrm{of} \mathrm{the}$ magnet three large concentric cylinders of soft steel were used as magnetic shields, and in addition the coils themselves were embedded in the two halves of a mass of Swedish iron $3 \times 5 \mathrm{cms}$. This combination gave a shielding effect of over 6000 times. Proportionality corrections were applied to all galvanometer deflexions.

It was necessary, in the process of measurement, to be able to reduce the sensitiveness of the bolometer-galvanometer combination in some known ratio. This was done by the use of four resistances which could be inserted separately in series with the galvanometer circuit, and which gave a graded reduction of sensibility of from $2 \cdot 9$ to 104.5 times.

Control and Operation.--The various parts of the apparatus were arranged to be controlled by the observer in front of the galvanometer scale. This was done by means of the five rods $r, r, r$, which operated respectively the spectrometer table, the sliding shade, the bolometer balancing arrangement, 
and the two shutters. An auxiliary rod also turned the micrometer screw of the polarizer mounting.

Supposing everything in adjustment, the process of making observations was fairly simple. The spectrometer having been set at the wave-length for which it was desired to measure the rotation, the sliding shade was moved by means of its screw adjustment till both beams were of equal intensity, as shown by the galvanometer returning to zero. The magnet-switch was then thrown, and in a few seconds the reading taken. The current was then reversed and the new reading noted, and so on until a set of four or five readings at regular intervals of perhaps 15 seconds, had been taken for each direction of the current. The difference between the means of each set of readings would give the quantity $d \mathrm{I}$, but it would also contain the small but still noticeable direct action of the magnet on the galvanometer. To eliminate this the galvanometer connexions were reversed by a suitable switch and the above process repeated, and a slightly different value for $d \mathrm{I}$ obtained. The mean of these two values gave the true one.

To measure the intensity I it was first necessary to reduce the sensibility by a considerable factor--usually $26^{\circ} \cdot 6$ times-by pulling one of the switches, $\mathrm{S} w$, thus inserting a series resistance. Then by closing each shutter separately two nearly equal values of $I$, or rather of $I / 26^{\circ} 6$, would be given, the mean of which was used. As explained above, the ratio $d \mathrm{I} / \mathrm{I}$ is proportional to the rotation occurring on reversal of the magnet.

By repeating this process for a dozen or twenty wavelengths distributed over the spectral region, in which it was possible to work, say from $\lambda=\cdot 6 \mu$ to $2 \cdot 2 \mu$, a very good rotation dispersion-curve could usually be obtained. As in the previous work, all the more careful mensurements had to be made at night.

Large and Small Rotations. - In the determination of large rotations this method has very evident limitations when compared with visual schemes, but as the largest measurable angle, perhaps $30^{\circ}$, is many times what would be met with in any work with films, this consideration is of no importance. Of more interest is the question of the smallest measurable rotation. This is largely a matter of the amount of energy incident on the bolometer strips available for measurement, and hence depends on the part of the spectrum; also on the general conditions of steadiness of the apparatus. No especial fains have ever been taken to actually determine the smallest rotation which could be detected, but in practice angles of 
less than $.003^{\circ}$ have frequently been measured, and it is believed that, with care, rotations of three or four, perhaps even of single, seconds could be detected.

\section{Preliminary Measurements.}

Calibration of Magnetic Field.- - It was desired to test the transmission and reflexion rotations of films under exactly the same conditions of field strength. Since they could not be located in the same part of the field for the two tests, this necessitated a calibration of the field. To do this a small flip-coil was used having the same area as that of the cross section of the beam as it passed through, or was reflected from, a film. From a large number of measurements curves were plotted showing the variation of field strength with position and with magnet current; and the current was accordingly adjusted in practice to give the same field strength whatever the position of the film.

Sensibility Reduction Factors.-As a very exact knowledge: of the reduction effect of the sories resistinces on the galvanometer-bolometer sensibility is required, this was measured in three ways:- by a step by step process; by the use of rotating sectors; and by computations from the results of mechanical rotations of the polarizer. Four resistances giving reduction factors styled A, B, C, and D respectively, were used ; and in the step by step way these factors were measured by comparing the effect on a certain galvanometer deflexion obtained, with full sensibility of the introduction of the resistance $A$. This was then similarly compared with $B$ and the process repeated for C and D. Very careful galvanometer proportionality corrections were required for the success of this method, and at best there are certain theoretical objections to it.

In the second way, by the aid of a rotating sector which transmitted only ${ }_{25}^{1}$ of the incident light the reduction factor C was measured by the comparison of two nearly equal galvanometer deflexions. For the energy transmitted by the rotating sector and measured with the highest sensibility gave almost the same throw as the total energy measured with the resistance $C$ in series. The values of $A$ and $B$ were likewise determined with the aid of suitable sectors.

By rotating the polarizer through small and accurately known angles, a third series of values was obtained, f'or these rotations were measured just as if they were of electromagnetic origin; and, by computing back, the necessary values to be assigned to the reduction factors were easily found. These values agreed very well with those determined by the last method, as may be seen from the following table. 


\section{TABLE I.}

Values of Sensibility Reduction Factors.
(1) Values given by step by step method .. 2.95
B.
(2) Measured with rotating sector ....... 2.90
$9 \cdot 45$
C.
$9 \cdot 20$
(3) Computed from rotations of the polarizer 2.92
$9 \cdot 15$
$26 \cdot 70$
Accepted values....... 2.90
$9 \cdot 20$
$26 \cdot 45$
$26 \cdot 60$

D, which was rarely used, was found by comparison with $C$ to be $\mathbf{1 0 4 . 5}$.

This table is of interest in that it gives an idea of the maximum accuracy which could be expected of this way of measuring magnetic rotations, or rather, of the largest error which is likely to enter from this source. It is true that sets (1) and (2) disagree by 2 per cent., and (1) and (3) by 3 per cent. ; but, as stated above, method (1) is not as free from objection as the other two, whose results differ by less than 1 per cent., and this is estimated as about the probable error. Correction for Errors due to Low Dispersion.-This was determined, as previously indicated, by comparison of the two curves made with the same rotating substance under the two different conditions of dispersion. As might be anticipated, the error in determining any particular rotation is dependent not oniy on the wave-length, that is, on the position of the point on the curve, but also on the actual form of the curve. Furthermore, the largest error is to be experted when we have the combination of an energy curve rapidly increasing-as the Nernst-glower energy curve in the early infra-red-and a rotation dispersion curve rapidly falling, as is the case for glass and similar substances.

This is well shown by the rotation dispersion curves of fluorite in $\mathrm{Pl}$. I. fig. 3 . The rotations on the upper curve are 30 per cent. higher at $65 \mu$, and the difference gradually lessens until they are equal at $2 \cdot 0 \mu$. An extrapolation to give the true form of tbe curve as determined with a spectrum of great dispersion would raise the upper curve by only about 1 per cent.

That the correction is much smaller, if not negligible, when the rotation increases with wave-length in the early infrared is indicated in Pl. I. fig. 4. The curve is for rotation on reflexion from a polished steel mirror in a field of 5700 units. It is essentially identical, though plotted some what differently, with the curve as measured for the same mirror with the earlier form of apparatus*. The corrections for a third type of curve-a horizontal straight line-could be deduced at once from the figures in Table II., and are negligible. With 
these three types it will be possible to estimate the corrections with ample accuracy for any of the curves discussed in the present paper. Most of them, as a matter of fact, are of the latter two types.

\section{Sources of Error. Accuracy of the Method.}

The writer has previously discussed $\dagger$ a number of sources of error incidental to this work. In the present case an attempt, at least, has been made either to remove these or make them subjects of special experimentation, with a view of determining the magnitude of the errors they introduce. It would seem then that the accuracy with which magnetic rotations can be measured in the infra-red is largely dependent on the care with which the preliminary experiments have been carried out.

Fortunately it is possible, as previonsly stated, to get a check on this accuracy by making known mechanical rotations of the plane of polarization and measuring them just as if of electromagnetic origin. Table II. shows the results of these tests. The rotation of the polarizer of $1^{\circ} \cdot 178-$ produced by six complete turns of the micrometer tangent-screw -was measured with the apparatus for a number of wavelengths and with each of the two sets of prisms and mirrors. Lest it be very reasonably objected that the mechanical rotations have been already used to determine a series of values of the reduction factors $A, B, C$, and $D$, - and that if these values are used the table would necessarily show a correspondence,- the series (2), determined by the preferable or rotating sector process, has been used in this computation.

TABLE II.

Test of Accuracy of Method by Measurement of a known Rotation of $1^{\mathrm{c}} \cdot 178$.

\begin{tabular}{|c|c||c|c|}
\hline Wave-length. & With $45^{\circ}$ prism. & Wave-length. & With $25^{\circ}$ prism. \\
\hline $.69 \mu$ & $* 1.191^{\circ}$ & $-59 \mu$ & $1 \cdot 166^{\circ}$ \\
.75 & $* 1.158$ & 64 & 1.160 \\
.81 & 1.170 & 72 & 1.170 \\
.88 & 1.163 & .85 & 1.172 \\
.98 & 1.174 & 1.04 & 1.168 \\
1.23 & 1.169 & 1.31 & 1.170 \\
1.61 & 1.170 & 164 & 1.171 \\
1.84 & 1.169 & 1.97 & 1.173 \\
2.01 & 1.189 & & \\
\hline
\end{tabular}

* The smaller amount of energy makes measurements in the shorter wave-lengths less reliable.

† Phil. Mag. loc. cit. p. 51. 
It will be seen from the above table that the actual and computed rotations usually differ by 1 per cent. or less. This, then, may be taken as about the order of the outstanding probable error of rotation measurements in this work. It is about as great an accuracy as may be reasonably expected in almostany spectro-bolometric or radiation work, and is ample for the present investigation.

In the matter of certainty of wave-length, as great accuracy cannot be claimed as might be expected in radiation problems where a spectrum of large dispersion and a very narrow bolometer-strip can be used. The spectrometer was calibrated in the present case by double dispersion tests, using a rocksalt prism on the second spectrometer to fix the wave-length scale. Each of the two prisms was tested several times in this way. For the $25^{\circ}$ prism the estimated probable error rises from a few thonsandths of a micron in the visible and early infra-red to about $01 \mu$ at $\lambda=\{\cdot 1 \mu$, and is perhaps three or four times as much at $2 \cdot 3 \mu$. For the $45^{\circ}$ prism the probable error hardly exceeds $\cdot 02 \mu$ anywhere. It must be remembered, however, that the bolometer-strip covers a much greater width of spectrum than this ; hence the determination of a rotation dispersion curve which had sharp bends or dips corresponding to sharp absorption-bands would be practically impossible.

\section{Part Ji.-Rotation Dispersion Curves for the Faraday and Kerr Effects.}

Deposition of Films.--The films were deposited cathodically on pieces of microscope cover-glass in an atmosphere of hydrogen, an induction-coil being used as a source of current and a small spark-gap introduced in the secondary circuit to make it practically one directional. A flat coil of pure iron wire, making a disk of 2.5 cms. diam., served as a cathode, and the best results were obtained with a vacuum of a fraction of a millimetre, and with the pieces of cover-glass just outside the cathode dark space and about $1 \mathrm{~cm}$. fiom the cathode. It was possible to use thin microscope-cover glass as a backing for the films, in spite of its poor optical surfaces; for it will be noted that in all cases the film was placed where the beam of light converged to a focus. Hence the loss of light occasioned by the departure from planeness of the surfaces was immaterial, while the advantage of having unly a small thickness of glass is evident.

Considerable care was required to secure films which were sufficiently uniform over the required area of $4 \times 15 \mathrm{mms}$, and free from oxidation. With the first induction-coil used- 
one furnishing a bigh voltage and small current-it was almost impossible to avoid a certain amount of oxidation. This gave place to a smaller coil, furnishing a larger current and taking a much shorter time-half an hour or less-to deposit a film. Still later a 2000 volt generator set was used, but most of the films discussed in the present paper were deposited with the smaller induction-coil.

Oxidation, when present, was readily detected by the brownish colour imparted to the film. It was doubtless caused by the minute quantity of occluded air which would still remain even after repeated washings with hydrogen. When, however, the greatest care was taken in getting rid of this remaining air by frequent washings with dry hydrogen, between short periods of electrical discharge, films were obtained which were quite colourless, of a greyish appearance when thin, and looking like smoked glass when thick. This accords with the description of Houllevigue * of the appearance of iron films free of oxidation. The films were kept in an atmosphere of dry hydrogen when not being used, and did not deteriorate appreciably for many weeks. When left for some time in moist air they slowly oxidized to a yellow colour.

Measurements.-About forty films were deposited in all, in lots of two or three at a time usually, and perhaps half of these tested. Out of this number four have been selected which had suitably varying thicknesses and which showed almost no traces of oxidation; and the curves made from these are show' in Pl. I. figs. 6-9.

FIG. 5.

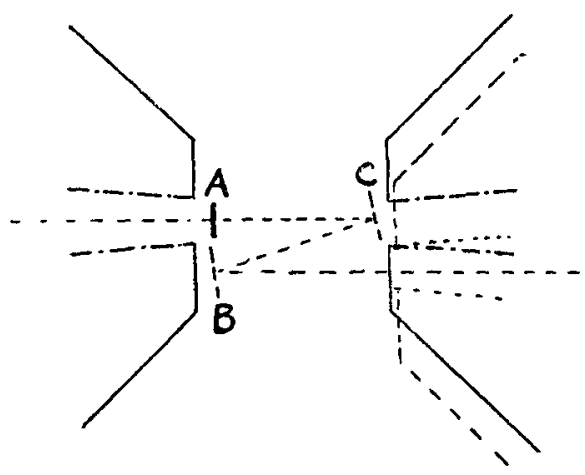

The rotations shown in the "transmission" curve were measured with the film in position $A$, fig. 5, the pole-pieces

* Jour. d. Phys. (4) iv. p. 406 (1905). 
being in line. The field was approximately 5700 c.G.S. units with a magnet current of 16 amps.

For the "reflexion" curve the film was held in position B and a piece of plane silvered glass placed at $C$. The beam striking the silver surface would be reflected to the film and from this through the other pole-piece, which was thrown out of line to the dotted position to allow the passage of the beam: it would then follow practically the same path as in the first case. The reflexion at the silver surface did not affect the polarization of the beam and the departure from normal incidence on the film-about $8^{\circ}$-was well within the limits of $15^{\circ}$, for which, as shown by Righi ${ }^{*}$, the rotation is practically the same as for normal incidence. This arrangement, then, served the purpose of the customary optical system in which the normally reflected beam is turned aside by a plane glass plate at $45^{\circ}$; such an arrangement would be of difficult, if not wholly impossible, application in the present problem. With a magnet current, of $13 \cdot 7$ amps., the field in this position was identical with that in position A.

Corrections. - Following the practice of Kundt, the rotations given by the curves and tables of the present paper are for the reversal of the magnetic field, unless otherwise specified, and hence are really doubled values. They were all determined with the use of the $25^{\circ}$ prism, and therefore, as has been noted in Part I., some correction may be necessary to the measured values. But it will be observed that the curves may be considered as partly straight lines and partly of the type of Pl. I. fig. 4; and in either case the corrections are negligible.

For the transmission curves, correction has been made for the rotation due to the glass on which the films were deposited. This was small-as the glass was only $\cdot 18$ to $\cdot 22 \mathrm{~mm}$. thick,but by no means negligible, for in some cases it may be comparable with the rotation of the film itself. 1t was carefully determined for selected sample pieces (correction being made for the use of $25^{\circ}$ prism as indicated in PI. I. fig. 3), and in each case the correction applied was proportional to the thickness of the particular piece of glass on which the film was deposited. In Pl. I. figs. 6-9 the dotted curves give the rotations as measured while the solid line curve has been corrected for the glass rotation. The curve for the glass alone is shown in Pl. I. fig. 6.

To be certain that no other influence than the magnetized film was causing rotation, trials were occasionally made for rotation on reflexion from two silver surfaces, instead of one

* Ann. Chim. Phys. (6) ix. p. 132 (1886). 
silver and one iron. Such rotations were always found to be extremely small. One has been plotted in PI. I. fig. 6, and denoted by the letter $\mathrm{S}$. It amounts to only about $\cdot 002^{\circ}$.

It is undoubtedly true that the results as given for the thinner films are somewhat in error because of multiple internal reflexions, but it would be hopeless to try to allow for these until we can separate more clearly the two rotation effects and perhaps localize the reflexion rotation. This error is not considered of importance except in the case of the very thinnest films. It is also a fact, from the work of previous observers *, that transmitted light from a magnetized film is slightly elliptically polarized. This would not cause any error in the present work; the rotation measured would be that of the major axis of the resulting ellipse.

Absorption Measurements. - It was thought to be of interest to determine if the absorbing and reflecting powers of the films showed a variation with wave-length corresponding to the rotation. Accordingly measurements were made of the transmission of each film, as well as of the reflecting power (by comparison with silver) of the front and rear surfaces. The curves for several films are shown in Pl. I. fig. 10, where T, the transmission, is approximately equal to $e^{-a t}, \alpha$ being the absorbing power and $t$ the thickness. No attempt has been made to correct for the complicated system of internal reflexions : this is in itself an extremely difficult problem for films as thin as some of these. The values of $T$ have been obtained by merely dividing the fraction of radiation transmitted by $1-R$, where $R$ is the reflecting power of the air-film surface. While not possessing the accuracy of the rotation measurements they serve very well to indicate the selective or non-selective character of the absorption or reflexion, which is the only part in which we are particularly interested.

It will be noted that the transmission-and therefore also absorption-for the pure iron films 15,22 , and 24 is almost non-selective or independent of wave-length. The same is true for film 17 whose curve is not shown. This explains the greyish colours of these films, and, in agreement with the conclusions of other observers as already pointed out, is accepted as evidence of their freedom from oxidation.

The curve of reflecting power with wave-length for film 15 is incidentally shown in Pl. I. fig. 13. It shows a slight increase of reflecting power in the infra-red, as is the case with pure iron in the mass $\dagger$. Film 17 shows a slightly greater increase

* See Harris, Phys, Rev. xxiv. p. 337 (1907.)

† Hagen and Rubens, Ann. d. Phys. viii. p. 1 (1902). 
of reflecting power with increasing wave-length, while films 22 and 24 show a somewhat greater decrease, but on the whole the reflecting power for the pure films is also relatively non-selective.

Thickness of Films.-The absorption and reflexion data would afford a possible means for determining the relative thicknesses of the various films; but, as Houllevigue and Passa * have shown, it is more accurate to compute the thicknesses from the rotation produced, since this is proportional to the thickness. Accordingly the thicknesses of the four films already mentioned, $15,17,22$, and 24 have been computed in this way, assuming Houllevigue and Passa's data and iron cathode films, as approximately $58 \mu \mu, 30 \mu \mu, 13 \mu \mu$, for $8 \mu \mu$ respectively $\dagger$. Kundt's results for films by electrolysis would give thicknesses about one third smaller.

Very Thin Films.- Tests were made also on a number of very thin films, some of them less than $0.5 \mu \mu$ in thickness, but the results are of questionable value, particularly those for rotation on reflexion. For in such thin films reflexion would take place not only at the first or air-film surface, but also at the film-glass boundary and at the glass-air or rear surface, and, because of the small loss on transmission, these three surfaces-or at least the first and last-would each return about the same amount of radiation. The latter part was generally excluded by covering the rear glass surface with an absorbing mixture of the same optical density as the glass, $i$. e. Canada balsam and lamp-black; but there would seem to be no way of separating the first two parts. In one case there was tried the experiment of depositing a thick film of platinum, and on this a thin film of iron. The rotation shown on reflexion was positive, as might be expected, due to the preponderance of the rotation of the doubly transmitted part of the beam. The only striking feature of the experiment was the enormously varying reflecting power shown by such a film, increasing from almost nothing in the visible to about 50 per cent. at $\lambda=2 \mu$.

In general, the results on very thin films indicated a somewhat larger reflexion rotation than the transmission. The latter rotation sometimes measured not more than $\cdot 003^{\circ}$, with the reflexion rotation perhaps twice as large and frequently appearing positive in the early infra-red.

Effect of Oxidation.-Considerable data on this point have

* Comptes Rendus, cxli. p. 29 (1905).

$\dagger$ This scale of thickness has been checked to within a few per cent. by subsequent measurements, by interference methods, of films deposited on optical glass. See "Addendum." 
been collected, but the results on different films are somewhat at variance. This is in accordance with the observations of Kundt*, who found very complicated effects when the mirror for which he was measuring the Kerr rotation was oxidized. In general it was found, however, that films of a reddish or brownish colour-indicating more or less oxidation-gave curves similar to those shown in Pl. I. figs. 11, 12, and 13. The difference between these and the curves for the pure iron films is seen in the rapid decrease of rotation with wave-length after the early infra-red. This is particularly true for the case of rotation on transmission, and was very marked in a film deposited in air instead of hydrogen, and which was presumably completely oxidized. Furthermore, the curves for rotation on transmission and reflexion do not show the same similarity as in the case of pure iron, and indeed in two of the cases shown the reflexion rotation appears positive for the shorter wave-lengtlis.

The change in the character of the rotation curves is seen to be accompanied by a marked variation of the transmission with wave-length, as shown by the dotted curves of Pl. I. fig. 10. For the opaque partially oxidized film 29 of Pl. I. fig. 13 only the reflexion rotation and reflecting power could be measured, but each is seen to change markedly with change of wave-length. As to the effect of uxidation on the magnitude of the rotation, films 7 and 14 show smaller rotations than the pure iron films, although film 14 has about the same absorption as film 22 . The reflexion rotation from film 29, however, is slightly greater for the early infia-red than that from film 15 or that due to a steel surface as shown in Pl. I. fig. 4.

Discussion of Results.- The first fact worthy of note which may be deduced from the curves is that the magnetic rotation, either on transmission or reflexion, of iron, instead of increasing indefinitely with wave-length, as might be concluded from observations in the visible spectrum, reaches a maximum value shortly outside the limit of this spectrum. This maximum rotation, which is reached for pure iron at about $\lambda=1.5 \mu$, is seen to be on the average a little over one and two-thirds times that for the wave-length of sodium light.

In the second place it will be noted that while the Faraday rotation decreases by five times in going from film 15 to film 24, the Kerr rotation is only halved. In other words the reflexion rotation increases less rapidly than the thickness of the film and soon reaches a maximum value. That this has been very nearly reached in film 15 is indicated by the

* Wied. Ann. xxvii. p. 199 (1886). 
agreement in magnitude of the rotations with those obtained with the polished steel surface, as well as the agreement with the results of other observers of the Kerr effect-working visually-reduced for this same field strength, viz. 5700 units. Moreover some later tests made visually with the aid of an ordinary half shade polarimeter on an opaque unoxidized film gave a rotation for sodium light, almost identical with that of this film. The seat of the Kerr rotation is unquestionably a thin surface-layer, and the foregoing results allow one to say that a layer of less than perhaps $20 \mu \mu$ thickness contributes considerably more than half of this rotation *. This is of the same order of magnitude as the thickness of the frequently assumed "transition layer" of optical theory.

A third point is the great similarity, if we confine our attention to the results for pure iron, of the curves for the two cases of rotation. While for the thinner films the Kerr rotation curve shows a more rapid decrease on the short wave-length side, the forms of the two curves for film 15 are almost identical-at least until beyond $1 \cdot 8 \mu$-as is shown by the following Table III. The rotation for the wave-length of sodium light is taken as unity in each case.

\section{TABLE III.}

Rotation dispersion of the Faraday and Kerr effects for a pure iron film (15).

\begin{tabular}{|c|c|c|c|c|c|}
\hline $\begin{array}{l}\text { Wave- } \\
\text { length. }\end{array}$ & $\begin{array}{c}\text { Transinission } \\
\text { rotation. }\end{array}$ & $\begin{array}{l}\text { Reflexion } \\
\text { rotation. }\end{array}$ & $\begin{array}{l}\text { Ware- } \\
\text { length. }\end{array}$ & $\begin{array}{c}\text { Transmission } \\
\text { rotation. }\end{array}$ & $\begin{array}{l}\text { Reflexion } \\
\text { rotation. }\end{array}$ \\
\hline $\begin{array}{c}\cdot 589 \mu \\
.70 \\
.80 \\
.90 \\
1 \cdot 00 \\
1 \cdot 10 \\
1 \cdot 20 \\
1 \cdot 30\end{array}$ & $\begin{array}{l}1 \cdot 00 \\
1 \cdot 20 \\
1 \cdot 33 \\
1 \cdot 44 \\
1 \cdot 52 \\
1.59 \\
1 \cdot 64 \\
1 \cdot 68\end{array}$ & $\begin{array}{l}-1.00 \\
-1.16 \\
-1.34 \\
-1.42 \\
-1.50 \\
-1.56 \\
-1.62 \\
-1.66\end{array}$ & $\begin{array}{l}1 \cdot 40 \\
1.50 \\
1 \cdot 60 \\
1.70 \\
1.80 \\
1.90 \\
2 \cdot 00 \\
2 \cdot 10 \\
2 \cdot 20\end{array}$ & $\begin{array}{l}1.71 \\
1.73 \\
1.73 \\
1.73 \\
1.71 \\
1.70 \\
1.68 \\
1.65 \\
1.62\end{array}$ & $\begin{array}{l}-1.70 \\
-1.74 \\
-1.74 \\
-1.76 \\
-1.76 \\
-1.76 \\
-1.76 \\
-1.76 \\
-1.76\end{array}$ \\
\hline
\end{tabular}

It would seem from the above that any explanation of the Kerr effect must indicate, more clearly than it does at present, its very close connexion with the Faraday rotation ; but unfortunately for the generality of this statement the

* We might also say that practically the full value is given by a layer $60 \mu \mu$ thick, which is about the same thickness as that found by Koenigsberger and Bender (Ann. d. Phys. xxvi. p. 763, 1908) for platinum and gold films which give the same phase change on reflexion as the massive metal. 
results for partially oxidized films show a considerable difference between the two curves. Kundt explained the varying effects obtained when the steel mirror for which he was measuring the Kerr effect was slightly oxidized, by the mutually interfering action of the two beams, one reflected from the air-oxide surface, and the other reflected and rotated by the oxide-metal surface. In the present case, however, we are dealing with a homogeneous layer, not a film of oxide on the metal, and the discrepancies cannot be as easily explained away.

The resemblance between the reflexion rotation curve for film 29 of Pl. I. fig. 13 and that obtained with a steel mirror, Pl. I. fig. 4, would indicate that the latter, though polished brightly, still had more or less oxide on its surface, perhaps in the minute scratches left by polishing. In the previous work on the Kerr effect (Phil. Mag. l. c.) the rotation dispersion curves for polished surfaces of nickel and cobalt showed this decrease of rotation with wave-length after about $1 \mu$, as well as steel. It remains to determine if cathodic surfaces of these metals show a similar effect. If, as shown above, the Kerr rotation takes place in an extremely thin surface film, it is not remarkable if polished surfaces fail to give results identical with those from the more perfect cathodic surfices.

Some such connexion as is seen to exist between rotatory power and absorbing or reflecting power might reasonably be expected. Thus in film 29 a rotatory power decreasing with wave-length is accompanied by an increasing reflecting power. The readiest explanation of this-overlooking for the moment the somewhat different state of the iron-would be that the $\mathrm{Kerr}$ effect is a function of the penetration of the wave on reflexion, and this may on the whole be less for a greater reflecting power. Similarly for the pure films, the flat part of the rotation dispersion curve accompanies a non-selective absorption or reflexion.

But this does not account in either case for the rapid change of rotatory power with wave-length in the visible and early intra-red spectrum. This accords with the conclusions reached by the writer from a study of the Kerr rotation curves for mirrors of steel, cobalt, and nickel, that this spectral region, - viz. from perhaps $\lambda=\cdot 4 \mu$ to $1 \mu$-has much the same optical significance in the case of metals that a region of rescnance absorption has for a dielectric. $\Lambda \mathrm{s}$ is well known, the refrictive indices of metals increase with wave-length in the visible spectrum. It remains to investigate their optical constants for the remainder of this region 
and as far as possible in the infra-red, for it quite possible that the anomalous character of the dispersion may cease, or at least change-as it does for the rotation dispersion-within the region in which it is possible to study it. Experiments to this end are now in progress.

\section{Part III.-Dependence of the Kerr Rotation on the Refractive Index of the Overilying Medium.}

In connexion with the foregoing experiments tests were made for several films of the rotation from the rear, or glassmetal, surface, and the unexpected result was found that the rotation in this case was more than half again as large as that for the ordinary air-metal surface. Thinking that some connexion might be established between the reflexion rotation and the optical density of the overlying medium, experiments were tried with fllms whose surfaces were covered with thin layers of various liquids, held in place and protected by a slip of microscope cover glass. The rotations measured in this way all proved larger than for the bare surface and by a factor not far different, in most cases, from the refractive index of the liquid.

It can be readily seen that the rotation as measured by the bolometric method for such cases as these, will not represent, without suitable corrections, the actual rotation which takes place at the liquid metal surface. For in the first place a part of the energy would be reflected from the surface of the glass cover slip and would be returned without undergoing any rotation. A smaller part would likewise be reflected from the glass-liquid surface, while the part which is reflected and negatively rotated by the true liquid-metal surface must also be affected by the positive rotation due to the double passage through glass and liquid. The rotation due to the latter need not be considered since the layer of liquid is so thin, but that due to the former must be corrected for. If we are dealing with rotation from the glass-metal surlace of the film the corrections are of the same sort, but slightly simpler, since the liquid is lacking. To make the first correction, that is, for the reflected energy which does not penetrate to the metal surface, we must know the combined reflecting power R, of the air-glass, glass-liquid, and liquid-metal surfaces, and also $R^{\prime}$ of the air-glass and glass-liquid surfaces alone. The former is obtained by measurement, the latter by calculation from the known refractive indices. Then of every 
$R$ parts of energy which are reflected and go to make up the measured quantity $I, R^{\prime}$ parts are inactive, having suffered no rotation and hence should be subtracted from $I$ in computing the actual rotation. This is equivalent to multiplying the rotation, measured in the ordinary way as $d \mathrm{I} / \mathrm{I}$, by $\mathrm{R} /\left(\mathrm{R}-\mathrm{R}^{\prime}\right)$.

When there is added to this the doubled rotation due to the passage twice through the glass the corrected rotation is obtained. To take the first case in Table IV. as an example,

\section{Table IV.}

Reflexion rotations from the rear (glass-metal) surfaces of pure iron films $15,17,22$.

\begin{tabular}{|c|c|c|c|c|c|c|}
\hline$\lambda$. & $\begin{array}{l}\text { Measured } \\
\text { rotation. }\end{array}$ & R. & $\mathbf{R}^{\prime}$. & $a^{\prime}$. & $\begin{array}{c}\text { Double } \\
\text { gluss } \\
\text { rotation. }\end{array}$ & $\begin{array}{l}\text { Corrected } \\
\text { rotation. }\end{array}$ \\
\hline \multicolumn{7}{|c|}{ Film 15.} \\
\hline $\begin{array}{l}\cdot 63 \mu \\
.74 \\
.94 \\
1.28 \\
1 \cdot 71 \\
2 \cdot 14\end{array}$ & $\begin{array}{l}-.262^{\circ} \\
-.348 \\
-. \cdot 480 \\
-.610 \\
-.662 \\
-.656\end{array}$ & $\begin{array}{l}.321 \\
.328 \\
.340 \\
.335 \\
.335 \\
.336\end{array}$ & $\begin{array}{l}\cdot 040 \\
\cdot 040 \\
\cdot 039 \\
\cdot 039 \\
\cdot 038 \\
\cdot 038\end{array}$ & $\begin{array}{l}-.299^{\circ} \\
-.396 \\
-.543 \\
-.691 \\
-.747 \\
-.740\end{array}$ & $\begin{array}{l}\cdot 143^{\circ} \\
\cdot 108 \\
\cdot 070 \\
\cdot 037 \\
\cdot 019 \\
\cdot 009\end{array}$ & $\begin{array}{l}-.442^{\circ} \\
-.504 \\
-.613 \\
-.728 \\
-.766 \\
-.749\end{array}$ \\
\hline \multicolumn{7}{|c|}{ Film 17.} \\
\hline $\begin{array}{c}\cdot 65 \mu \\
\cdot 75 \\
.96 \\
1 \cdot 30 \\
1 \cdot 74 \\
2 \cdot 16\end{array}$ & $\begin{array}{l}--.211^{\circ} \\
-.312 \\
-.414 \\
-.517 \\
-.570 \\
-.567\end{array}$ & $\begin{array}{l}\cdot 257 \\
\cdot 259 \\
\cdot 247 \\
\cdot 229 \\
\cdot 215 \\
\cdot 191\end{array}$ & $\begin{array}{l}\cdot 040 \\
.040 \\
.039 \\
\cdot 039 \\
\cdot 038 \\
\cdot 038\end{array}$ & $\begin{array}{l}-\cdot 250^{\circ} \\
-\cdot 369 \\
-\cdot 492 \\
-\cdot 624 \\
-\cdot 693 \\
-\cdot 708\end{array}$ & $\begin{array}{l}\cdot 133^{\circ} \\
\cdot 100 \\
\cdot 066 \\
\cdot 033 \\
.016 \\
\cdot 009\end{array}$ & $\begin{array}{l}-.383^{\circ} \\
-.469 \\
-.558 \\
-.657 \\
-.709 \\
-.717\end{array}$ \\
\hline \multicolumn{7}{|c|}{ Film 22.} \\
\hline $\begin{array}{c}\cdot 66 \mu \\
\cdot 75 \\
.96 \\
1 \cdot 30 \\
1 \cdot 74 \\
2 \cdot 16\end{array}$ & $\begin{array}{l}-.084^{\circ} \\
-.181 \\
-.314 \\
-.414 \\
-.459 \\
-.430\end{array}$ & $\begin{array}{l}.090 \\
.088 \\
.080 \\
.081 \\
.075 \\
.070\end{array}$ & $\begin{array}{l}.040 \\
.040 \\
.039 \\
.039 \\
.038 \\
.038\end{array}$ & $\begin{array}{l}-.152^{\circ} \\
-.332 \\
-.613 \\
-.798 \\
-.930 \\
-.940\end{array}$ & $\begin{array}{l}\cdot 133^{\circ} \\
\cdot 100 \\
\cdot 066 \\
\cdot 033 \\
\cdot 016 \\
\cdot 009\end{array}$ & $\begin{array}{l}-.285^{\circ} \\
-.432 \\
-.679 \\
-.831 \\
-.946 \\
-.949\end{array}$ \\
\hline
\end{tabular}

the measured rotation $(=45 / \pi d \mathrm{I} / \mathrm{I})$ for $\lambda=\cdot 63 \mu$ was found to be $-262^{\circ}$. The reflecting power of the glass and glassmetal surfaces was $\cdot 321$ and that of the glass alone (there being no liquid in this case) was $\cdot 040$. This gives a correcting 
factor of $\cdot 321 / \cdot 281$ which hrings the measured rotation up to $\alpha^{\prime}-299^{\circ}$. Subtracting from this the glass rotation of $+\cdot 143^{\circ}$ we get the corrected rotation of $-442^{\circ}$. The process is entirely similar for the case when the liquid is used, save that the glass reflecting power is made slightly largerperhaps $\cdot 045$ - to take account of the additional, or glassliquid, surface. The cover slips used were of practically the same thickness as the glass on which the tilms themselves were deposited and hence the glass rotation was the same in either case.

The corrected rotations from the above table have been plotted in the curves of Pl. I. fig. 14. As in all the cases considered these are the doubled rotations occurring on reversal of magnetization. It will be noted that the curves have the same general form as those of the Kerr effect in Pl. I. figs. 6-8, but that the rotations are all considerably larger. The remarkable feature, however, is that the magnitude of the rotations is nearly the same for the three films of very different thicknesses, and that the thinnest film shows the largest rotation.

In the following Table V. (p. 98) the results are shown of a number of experiments with liquids of optical densities ranging from 1.33 to 1.72 .

Discussion of Results. - In Voigt's * recent " Magneto- und Electrooptik," which contain the most complete treatment of the Kerr phenomenon that has yet appeared, there is reached on p. 316 an expression for the rotation on normal reflexion and for polar magnetization-the case we are considering - which may be written

$$
\text { Rotation }=x=\left|\frac{Q(k-i)}{n\left(1+k^{2}\right)}\right|,
$$

where $Q$ is a complex which does not contain $n$, and $n$ and $k$ are respectively the refractive index and absorption index of the metal. The equations have not been worked out for the case of a surrounding medium other than vacuum or air, and until this has been done it is useless to attempt any satisfactory justification of the above results on theoretical gronnds. But it is of interest to note that if we grant two not unreasonable assumptions +- viz. that the refractive index

* W. Voigt, Magneto- und Electrooptik, Leipzig, 1908.

+ Experiments now in progress in this laboratory show that the reflecting powers of metals, when overlaid with various liquids, are in agreement with the well-known formulæ for metallic reflexion, if assumptions similar to these are made. In this light they seem extremely reasonable.

Phil. Mag. S. 6. Vol, 18, No. 103. July 1909. 


\section{Table V.}

Showing effect on the Kerr rotation of overlaying iron surface with liquids of various refractive indices. $\alpha$ is the rotation due to the metal surface in air, and $\beta$ to the same surface overlaid with the liquid.

(1) With Potassium mercuric iodide solution. $N_{\mathrm{p}} 1 \cdot 72$.

Film. Wave-length. Rotation $\alpha$, Rotation $\beta . \quad \beta / a . \quad$ Mean.

\begin{tabular}{|c|c|c|c|c|c|}
\hline $15 \ldots \ldots \ldots$ & $\begin{array}{l}\cdot 84 \mu \\
1 \cdot 11 \\
1 \cdot 74\end{array}$ & $\begin{array}{l}-.357^{\circ} \\
-.420 \\
-.462\end{array}$ & $\begin{array}{l}-.651^{\circ} \\
-.748 \\
-\cdot 763\end{array}$ & $\begin{array}{l}1.82 \\
1.78 \\
1.65\end{array}$ & $1 \cdot 75$ \\
\hline $\begin{array}{c}16 \\
\text { (similar to } \\
15 \text { ). }\end{array}$ & $\begin{array}{l}\cdot 84 \mu \\
1 \cdot 11 \\
1.74\end{array}$ & $\begin{array}{l}-\cdot 355^{\circ} \\
-\cdot 435 \\
-\cdot 480\end{array}$ & $\begin{array}{l}-.659^{\circ} \\
-.780 \\
-.830\end{array}$ & $\begin{array}{l}1.85 \\
1.79 \\
1.73\end{array}$ & $1 \cdot 79$ \\
\hline$\underset{\text { (opaque) }}{29 . . . . . . .}$ & $\begin{array}{l}.67 \mu \\
.82 \\
1.07 \\
1.69\end{array}$ & $\begin{array}{l}-\cdot 388^{\circ} \\
-.403 \\
-\cdot 378 \\
-.245\end{array}$ & $\begin{array}{l}-.704^{\circ} \\
-.691 \\
-.632 \\
-.399\end{array}$ & $\begin{array}{l}1.81 \\
1.71 \\
1.67 \\
1.63\end{array}$ & 1.71 \\
\hline
\end{tabular}

(2) With Bromnaptbalin. $\mathrm{N}_{\mathrm{D}} \mathbf{1} \cdot 66$.

15

$\begin{array}{cccc}\cdot 84 \mu & -\cdot 357^{\circ} & -\cdot 630^{\circ} & 1 \cdot 76 \\ 1 \cdot 10 & -.420 & -.698 & 1.66 \\ 1.74 & -.462 & -\cdot 737 & 1.59\end{array}$

(3) With Canada balsam. $N_{D} 1 \cdot 55$.

15

$\begin{array}{ccccc}15 \ldots . . . . . . . & .84 \mu & -.357^{\circ} & -.593^{\circ} & 1.66 \\ & 1.10 & -.4 .0 & -.686 & 1.63 \\ & 1.74 & -.462 & -.753 & 1.63 \\ \begin{array}{c}\text { Polished } \\ \text { steel. }\end{array} & .84 \mu & -.385^{\circ} & -.504^{\circ} & 1.31 \\ & 1.41 & -.340 & -.452 & 1.33 \\ & 1.96 & -.261 & -.365 & 1 \cdot 40\end{array}$

$1 \cdot 64$

(4) With Glycerine. $\mathrm{N}_{\mathrm{D}} 1 \cdot 45$.

$\begin{array}{ccllll}15 \ldots \ldots \ldots . . & .84 \mu & -.357^{\circ} & -.556^{\circ} & 1.56 & \\ & 1.11 & -.420 & -630 & 1.50 & 1.50 \\ & 1.74 & -.462 & -.670 & 1.45 & \\ 29 \ldots \ldots \ldots \ldots & .67 \mu & -.388^{\circ} & -.596^{\circ} & 1.53 & \\ & .82 & -.403 & -.617 & 1.53 & 1.50 \\ & 1.07 & -.378 & -.557 & 1.47 & \\ & 1.69 & -.245 & -.364 & 1.48 & \\ \text { Polished } & .84 \mu & -.385^{\circ} & -.534^{\circ} & 1.39 & \\ \text { steel. } & 1.41 & -.340 & -.484 & 1.42 & 1.42 \\ & 1.96 & -.261 & -.379 & 1.45 & \end{array}$

(5) With Water. $\mathrm{N}_{\mathrm{D}} 1 \cdot 33$.

16

$\begin{array}{llll}.84 \mu & -.355^{\circ} & -\cdot 438^{\circ} & 1 \cdot 23 \\ 1 \cdot 11 & -.435 & -.523 & 1 \cdot 20 \\ 1.74 & -.480 & -.567 & 1 \cdot 18\end{array}$


$n$ may be taken as relative to that of the surrounding medium, while the absorption index $k$ is constant-we reach at once the conclusion from the above equation that the Kerr rotation should increase directly as the refractive index of the overlying medium.

That this is at least the general trend of the facts is shown by Table V. 'The agreement is by no means good, but, for the experiments with cathode films, the mean values of the ratios-if we allow that a mean has any significance when there is such a decided "drift" of the results-differ generally by only a few per cent. from the refractive indices. There is no evident explanation of the rapid decrease of this ratio with increasing wave-length; it is much greater than the decrease of index of the liquid would require it to be. The results with steel mirrors are seen to differ widely from those with cathode films, particularly in case (3) with Canada balsam as the liquid. But a polished metal surface, with all its microscopic scratches, is not to be compared for ultimate smoothness of structure with a cathode mirror on a glass surface. It may be indeed that the viscous Canada balsam did not penetrate to the bottom of crevices of the steel surface.

Passing to the case of rotation from the rear surfaces of films we should expect to find such rotations larger than those from the first surfaces by a fraction equal to the refractive index of the glass, in this case about $1 \cdot 49$. But if the curves of Pl. I. fig. 14 are compared with the corresponding ones of Pl. I. figs. 6-8, it will be seen that the rotations have increased from over 1.7 in film 15 to nearly 3 times in film 22 . Two explanations are offered for this: that the state of aggregation and physical properties of the metal are modified in the layer next the glass; or, more probably, that the matter is complicated by internal reflexions in the case of the thinner films.

This would possibly explain these results and at the same time not throw discredit on the work of Part II. where the reflected rotation is measured from the thinner films, because the reflecting power of a plain or air-iron surface is much greater than that of the glass-iron. Hence, since by Stokes' law these reflecting powers also hold for the internal reflexions, the amount of energy returned on the first internal reflexion is much less in the cases of Part II. than in the present experiments.

An attempt was made to verify qualitatively these conclusions as to the variation of the Kerr effect with the overlying medium, by means of visual observations with 2 $\mathrm{H} 2$ 
half shade polarimeter, using sodium light. An opaque film was deposited on plate glass and the Kerr rotation measured with, and without, the use of Canada balsam. The observations were very difficult and not very satisfactory in the latter case, but, when corrected for the doubled rotation of the glass cover slip, came out about half larger.

\section{Summary.}

1. The writer's bolometric method of measuring magnetic rotations has been developed until, under favourable circumstances, rotations of less than $001^{\circ}$ can be detected, and angles somewhat larger measured with an accuracy approximating 1 per cent. It is applicable to a range of spectrum from the $\mathrm{D}$ lines to $\lambda=2 \cdot 2 \mu$.

2. The dispersion of magnetic rotation for cases of reflexion from and transmission through, iron catbode films, has been measured. For the pure iron fllms these curves are entirely similar and show a gradual increase of rotating power until wave-length $1.5 \mu$ is reached, where the rotation reaches a maximum value over one and two-thirds times as great as that for the $\mathrm{D}$ lines.

3. After a certain thickness is reached the reflexion rotation ceases to increase further with thickness of film. A layer of iron, of thickness less than $20 \mu \mu$, or about that of the assumed "transition layer" of optical theory, gives rise to considerably more than half of this rotation.

4. Partially oxidized films do not show the similarity hetween the reflexion and transmission rotation dispersion curves that pure films do. The rotation for oxidized films reaches a maximum in the early infra-red and falls off rapidly with further increase in wave-length. This change in form of the rotation curve with oxidation is accompanied by a corresponding change in the transmitting or reflecting povers as a function of wave-length.

5. The Kerr rotation is nearly proportional to the refractive index of the overlying medium, which, with certain assumptions, may be shown to be in accordance with Voigt's theory.

Physical Laboratory,

University of Wisconsin, Oct. $\$ 1,1908$.

\section{Andendum.}

Since the above was written, Skinner and Tool's paper on the optical properties of magnetic films has appeared (Phil. Mag. Dec. 1908). It: is a valuable contribution to the literature of the subject, showing very clearly as it does the 
different types of films which may be produced by the cathode deposit : the writer, however, is obliged to differ from them in some of their conclusions.

A comparison of their work with the pre:ent shows at once that what are here referred to as "oxidized" films would seem to be identical with their "dark" films, and the "puro iron" films of the present work, while not produced in just the same way, have the general appearance and non-selective relecting and absorbing powers which they describe for their "metallic" type. The chief objection to calling the latter two virtually identical is the abnormally high rotating power (per unit thickness) and extinction coefficients which they assign to these "metallic" films. These are some three times larger than for electrolytic iron, while their determinations of these quantities for electrolytic films are considerably higher than those of other ohservers.

A possible explanation of this lies in their thickness measurements ; for their method, it is believed, results in a considerable underestimate of the thickness of the film and hence overestimate of its rotating power and extinction coefficient. Their method-an interference one-involves the assumption that the phase changes on reflexion at glass and iron surfaces are the same, and this they were able to justify for very thin films. But Koenigsterger and Bender (Ann.d. Phys. xxvi. p. 763, 1908) find for the cases of gold and platinum at least, that while this is true for extremely thin films it is by no means the case for thicker ones.

To test this for iron, a number of films were deposited on optical glass and their thicknesses measured for sodium light after the manner of Skinner and Tool. The thicknesses were then measured by the method of Patterson (Phil. Mag. ii. p. 652, 1902), whereby the effect of any possible phase change is eliminated by a second cathode deposit, and in every case this gave considerably larger results. The true thickness was from a fraction larger, to more than twice as great as that measured in the first way, and this is more than sufficient to explain the abnormal values above mentioned.

Since the magnetic rotation for sodium light as well as the thickness was measured for these latter films, data were obtained for checling the estimates of the thickness of the films used in Part II. of the present work. These were checked to within a few per cent., or within the error of measurement.

In regard to the yellow-brown or "dark" films, Skinner and Tool consider that the metal is bere in a different state 
of aggregation and deny the possibility of any chemical combination of the metal with a gas. The writer, on the other hand, has assnmed in common with Houllevigue, that such films are the result of partial oxidation. This view soemed to be supported by the fact, already mentioned, that films deposited in oxygen (air) showed the same decrease of rotation with increasing wave-length after about $\lambda=1 \mu$. To throw more light on this point, a thick film, giving a rotation (Na light) of $+2^{\circ} .5$ before oxidation, was oxidized by moist air and ozone and its rotation then measured bolometrically. When corrected for the glass, the results gave $+014^{\circ}$ for $\lambda=1.08 \mu$, and $+.006^{\circ}$ for $\lambda=1.75 \mu$, the successive measurements differing by only a few thousandths of a degree. It will be noted that the rotation is still in the same sense but many times smaller than before, and that it shows this decrease with increasing wave-length after $\lambda=1 \mu$. These same characteristics are exhibited, in a smaller degree, by the yellow-brown films.

In conclusion, it seems to the writer that it is unnecessary at present to explain either of the types of films obtained by the cathode deposit as an especially anomalous state of the metal. For the "metallic" type, as shown by Skinner and Tool, in general resembles, more or less, electrolytically deposited films; and in the case of the "dark" films, while it is admittedly difficult to account for the presence of oxygen in the depositing chamber when one notes the care taken by these experimenters, these films show at least some of the characteristics of oxides.

X. On the Amount of Radium present in Sea-Water. By A. S. Eve, M.A., D.Sc., McGill University, Montreal*.

$\mathrm{T}$ this paper measurements will be expressed in terms of 1 billionths $\left(10^{-12}\right)$ gram of radium per kilogram of water tested.

Three observers have made determinations of the amount of radium in sea-salt or sea-water.

Strutt $\dagger$ found $7.5 \times 10^{-14}$ gram of radium per gram of sea-salt, and this is equivalent to $2 \cdot 3 \times 10^{-12}$ gram radium per kilogram of sea-water. 'This, the frst determination, was stated to be approximate only.

The writer $\ddagger$ examined Inagua sea-salt, and a sample of

* Communicated by the Author.

+ Proc. Roy. Soc. A. vol. lxxxviii. p. 151.

$\ddagger$ Phil. Mag. Feb. 1907. 
FiG. 3.-Curres for rotation on transmission through Fluorite,
showing effect of using high and low dispersion.

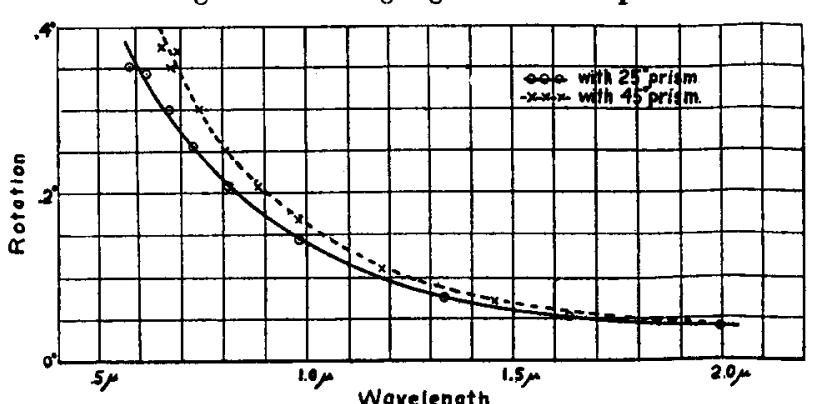

FIG. 4.- Curves for rotation on reflexion from steel, showing

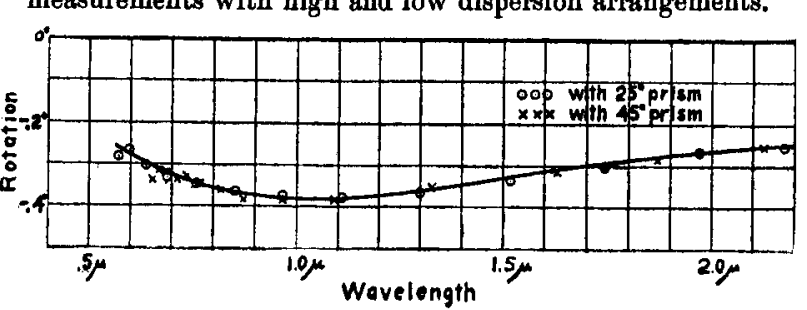

Fila. 6. - Curres for film 15, of $58 \mu \mu$ thickness.

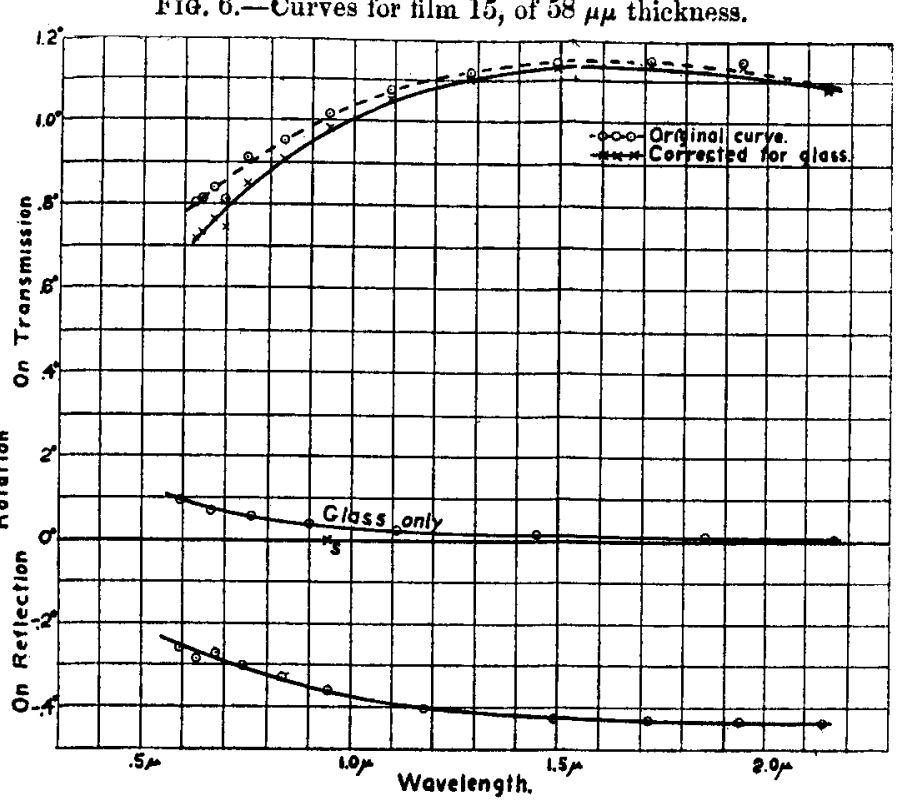

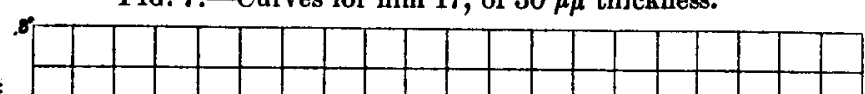

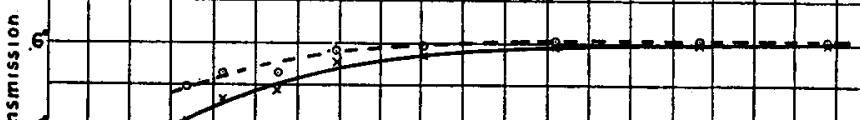

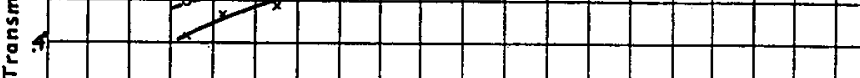

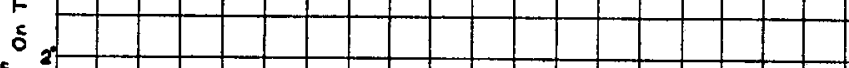
总

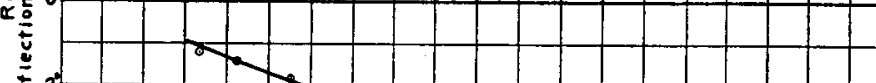

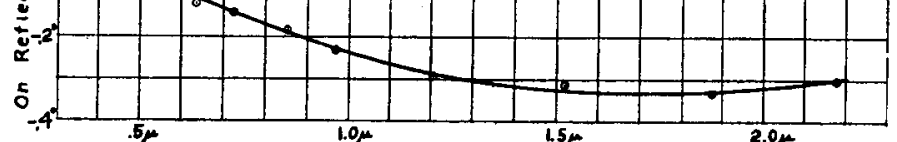

FIG.11.-Curves for partially oxidized film 7. Transmission curve refiexion curve, points plotted as triangles were deter-
mined with the rear glass surface covered with Canada
balsam and lamp-black.

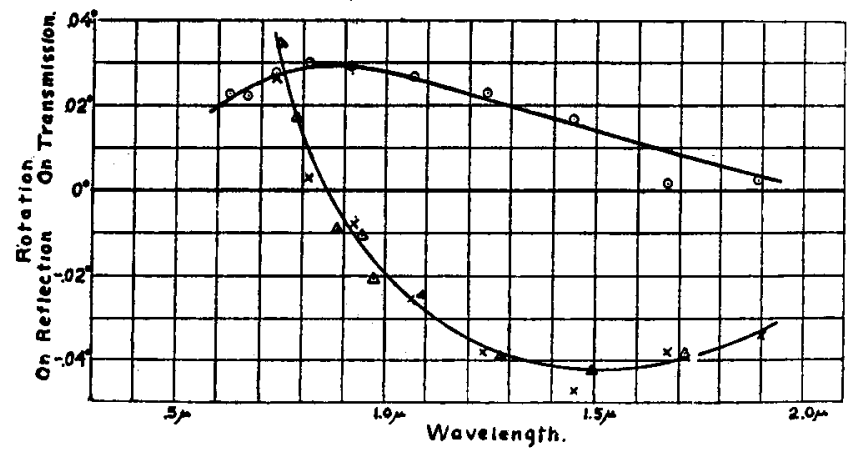

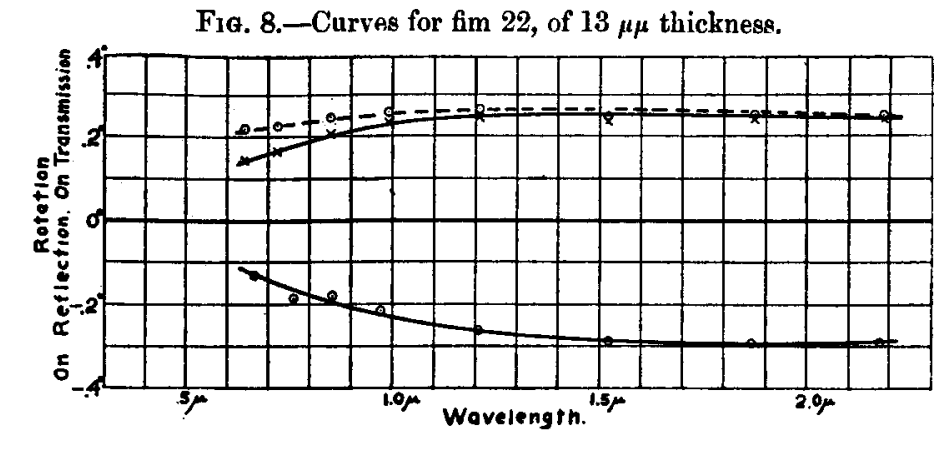

FIG. 12.-Curres for partially oxidized film 14. Transmission
curve has been corrected for rotation due to glass.

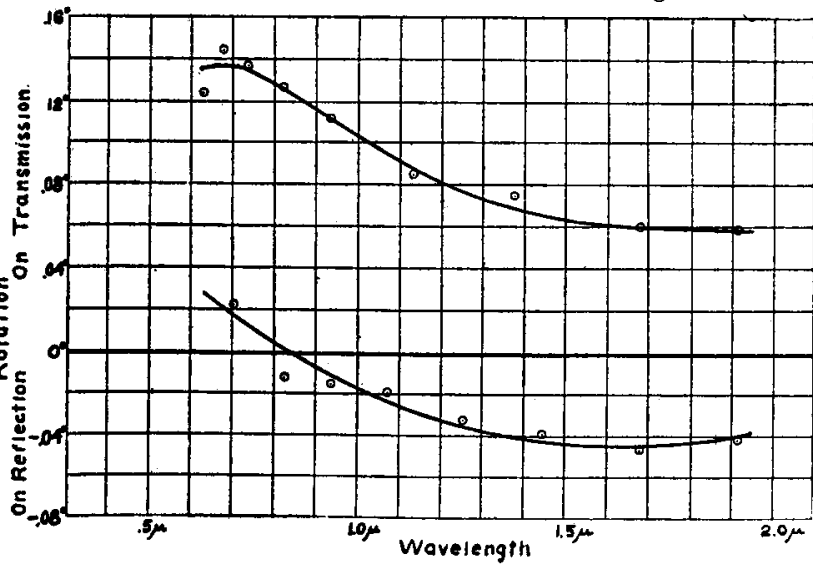

FIr. 9.-Curves for film 24 , of $8 \mu \mu$ thickness.

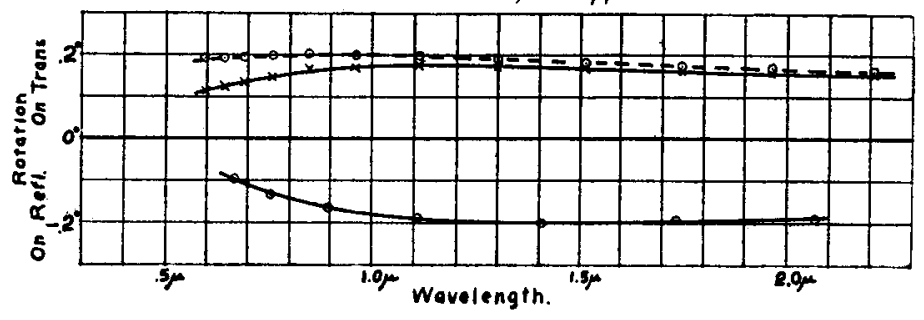

Fia. 13.-Curves for rotation on reflexion and for reflecting
power of opaque, partiully oxidized, film 29. The powfer of opaque, partially oxidized, film 29 .
for iower curve of pure iron film 15 is show

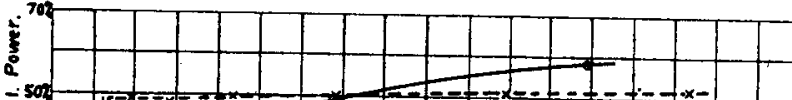
$a^{500}$

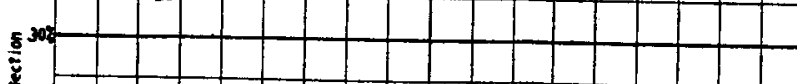

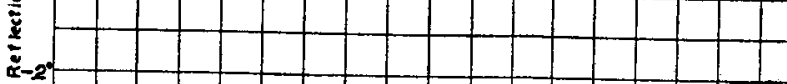
$+1+2+1+10$

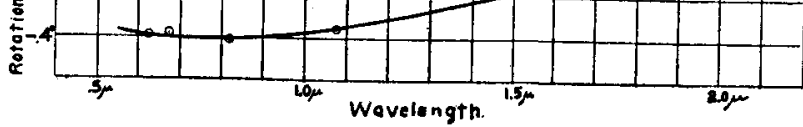

FIG. 10.-Curves showing the variation of transmission
with wave-length for the various films.

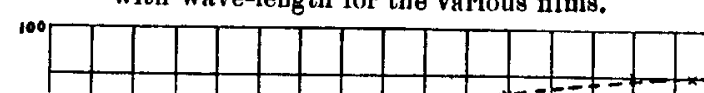

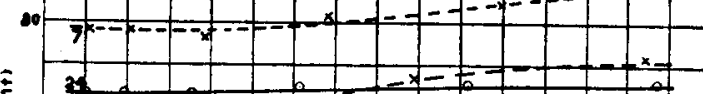
50000 (140-1

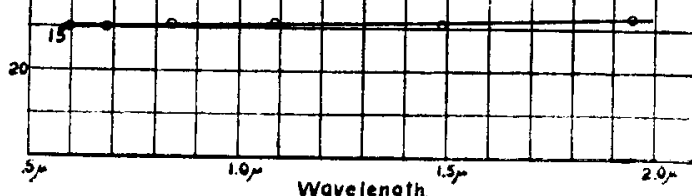

FIG. 14.-Curres showing the rotation on reftexion from the

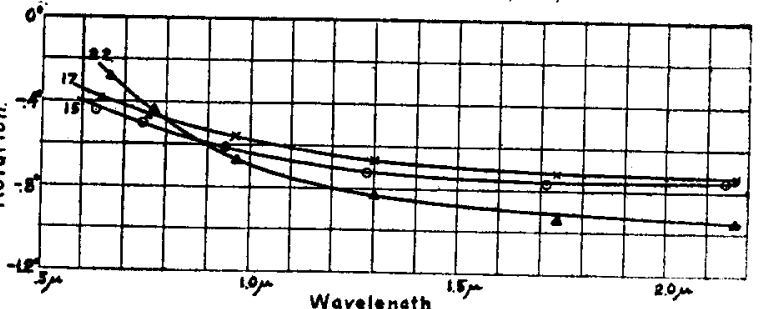

